\title{
Self-Dual Configurations in a Generalized Abelian Chern-Simons-Higgs Model with Explicit Breaking of the Lorentz Covariance
}

\author{
Rodolfo Casana ${ }^{1}$ and Lucas Sourrouille ${ }^{2}$ \\ ${ }^{1}$ Departamento de Física, Universidade Federal do Maranhão, 65080-805 São Luís, MA, Brazil \\ ${ }^{2}$ Universidad Nacional Arturo Jauretche, Florencio Varela, 1888 Buenos Aires, Argentina \\ Correspondence should be addressed to Rodolfo Casana; rodolfo.casana@gmail.com
}

Received 10 February 2016; Revised 11 July 2016; Accepted 26 July 2016

Academic Editor: Edward Sarkisyan-Grinbaum

Copyright ( 2016 R. Casana and L. Sourrouille. This is an open access article distributed under the Creative Commons Attribution License, which permits unrestricted use, distribution, and reproduction in any medium, provided the original work is properly cited. The publication of this article was funded by $\mathrm{SCOAP}^{3}$.

\begin{abstract}
We have studied the existence of self-dual solitonic solutions in a generalization of the Abelian Chern-Simons-Higgs model. Such a generalization introduces two different nonnegative functions, $\omega_{1}(|\phi|)$ and $\omega(|\phi|)$, which split the kinetic term of the Higgs field, $\left|D_{\mu} \phi\right|^{2} \rightarrow \omega_{1}(|\phi|)\left|D_{0} \phi\right|^{2}-\omega(|\phi|)\left|D_{k} \phi\right|^{2}$, breaking explicitly the Lorentz covariance. We have shown that a clean implementation of the Bogomolnyi procedure only can be implemented whether $\omega(|\phi|) \propto \beta|\phi|^{2 \beta-2}$ with $\beta \geq 1$. The self-dual or Bogomolnyi equations produce an infinity number of soliton solutions by choosing conveniently the generalizing function $\omega_{1}(|\phi|)$ which must be able to provide a finite magnetic field. Also, we have shown that by properly choosing the generalizing functions it is possible to reproduce the Bogomolnyi equations of the Abelian Maxwell-Higgs and Chern-Simons-Higgs models. Finally, some new self-dual $|\phi|^{6}$-vortex solutions have been analyzed from both theoretical and numerical point of view.
\end{abstract}

\section{Introduction}

A time ago it was shown that (1+2)-dimensional matter field interacting with gauge fields whose dynamics is governed by a Chern-Simons term supports soliton solutions $[1,2]$ (for a review see [3-7]). These models have the particularity to become self-dual when the self-interactions are suitably chosen [8-11]. When self-duality occurs the model presents interesting mathematical and physical properties; for example the second order Euler-Lagrange equations can be solved by a set of first-order differential equations $[12,13]$ and the model admits a supersymmetric extension [14]. The ChernSimons gauge field dynamic remains the same when coupled with matter fields either relativistic $[8,9]$ or nonrelativistic $[10,11]$. In addition the nature of the soliton solutions can be topological and/or nontopological [15].

The inclusion of nonlinear terms to the kinetic part of the Lagrangian has interesting consequences, as, for example, the existence of topological defects without a symmetrybreaking potential term [16]. In the recent years, theories with nonstandard kinetic term, named $k$-field models, have received much attention. The $k$-field models are mainly in connection with effective cosmological models [17-23], as well as the tachyon matter [24] and the ghost condensates [25-29]. The strong gravitational waves [30] and dark matter [31] are also examples of noncanonical fields in cosmology. The investigations concerning the topological structure of the $k$-field theories have shown that they support topological soliton solutions both in pure matter models and in gauged field models [32-49]. These solitons have certain features which are not necessarily shared with those of the standard models [50-52].

The aim of this paper is to study a Chern-Simons-Higgs model with a generalized dynamics which breaks Lorentz covariance; that is,

$$
\left|D_{\mu} \phi\right|^{2} \longrightarrow \omega_{1}(|\phi|)\left|D_{0} \phi\right|^{2}-\omega(|\phi|)\left|D_{i} \phi\right|^{2}
$$

The nonstandard dynamics is introduced by the functions $\omega_{1}$ and $\omega$, depending on the Higgs field. During the 
implementation of Bogomolnyi trick it is demonstrated that self-dual configurations exist if the function $\omega$ is proportional to $|\phi|^{2 \beta-2}$ with $\beta \geq 1$. On the other hand, the function $\omega_{1}$ remains arbitrary but near the origin should behave as $|\phi|^{2 \delta}$ with $\delta \geq-1$ in order to have a well behavior for the magnetic field. In particular we have chosen the functions $\omega_{1}$ and $\omega$, to be

$$
\begin{aligned}
\omega_{1}(|\phi|) & =|\phi|^{2 M}, \\
\omega(|\phi|) & =(N+1)|\phi|^{2 N},
\end{aligned}
$$

where $M \geq-1$ and $N \geq 0$. This way, the Bogomolnyi equations produce an infinite number of soliton solutions, one for each value of the pair $(N, M)$. It is possible to show that, for particular values of $N, M$, the Bogomolnyi equations of the Maxwell-Higgs or Chern-Simons Higgs models can be recuperated. Finally, we have constructed, analytically and numerically, novel soliton solutions for some values of $N$ and M.

\section{The Theoretical Framework}

Following the same ideas introduced in [32-49], we start by considering a generalized (2+1)-dimensional Chern-SimonsHiggs (CSH) model where the complex scalar field possesses a modified dynamic. Such a model is described by the following action:

$$
\begin{aligned}
S=S_{\mathrm{cs}} & +\int d^{3} x \\
\cdot & {\left[\omega_{1}(|\phi|)\left|D_{0} \phi\right|^{2}-\omega(|\phi|)\left|D_{i} \phi\right|^{2}-V(|\phi|)\right], }
\end{aligned}
$$

where $S_{\text {cs }}$ represents the Chern-Simons action given by

$$
S_{\mathrm{cs}}=\int d^{3} x \frac{\kappa}{4} \epsilon^{\mu \nu \rho} A_{\mu} F_{\nu \rho}
$$

The covariant derivative $D_{\mu} \phi$ is defined by

$$
D_{\mu} \phi=\partial_{\mu} \phi-i e A_{\mu} \phi,
$$

with $\mu=0,1,2$. The metric tensor is $g_{\mu \nu}=(1,-1,-1)$ and $\epsilon^{\mu \nu \rho}\left(\epsilon^{012}=1\right)$ is the totally antisymmetric Levi-Civita tensor.

In action (3) we notice the usual Higgs kinetic term, $\left|D_{\mu} \phi\right|^{2}=\left|D_{0} \phi\right|^{2}-\left|D_{k} \phi\right|^{2}$, was replaced by a more generalized term, $\omega_{1}(|\phi|)\left|D_{0} \phi\right|^{2}-\omega(|\phi|)\left|D_{k} \phi\right|^{2}$, which breaks explicitly the Lorentz covariance. The dimensionless functions $\omega_{1}(|\phi|)$ and $\omega(|\phi|)$ are nonnegative and, in principle, arbitrary functions of the complex scalar field $\phi$. The function $V(|\phi|)$ is a self-interacting scalar potential.

The gauge field equation obtained from the action (3) is given by

$$
\frac{\kappa}{2} \epsilon^{\mu \alpha \beta} F_{\alpha \beta}-e \mathcal{J}^{\mu}=0,
$$

with $\mathcal{J}^{\mu}=\omega_{1} \delta_{0}^{\mu} J^{0}+\omega \delta_{k}^{\mu} J^{k}$ being the conserved current of the model and $J^{\mu}=i\left[\phi\left(D^{\mu} \phi\right)^{*}-\phi^{*}\left(D^{\mu} \phi\right)\right]$ being the conventional current density. Similarly, the equation of motion of the Higgs field is

$$
\begin{aligned}
0= & \left(\partial_{0} \omega_{1}\right) D_{0} \phi+\omega_{1} D_{0}\left(D_{0} \phi\right)-\frac{\partial \omega_{1}}{\partial \phi^{*}}\left|D_{0} \phi\right|^{2} \\
& -\left(\partial_{k} \omega\right) D_{k} \phi-\omega D_{k}\left(D_{k} \phi\right)+\frac{\partial \omega}{\partial \phi^{*}}\left|D_{k} \phi\right|^{2}+\frac{\partial V}{\partial \phi^{*}} .
\end{aligned}
$$

From (6), the Gauss law reads

$$
\kappa B=e \omega_{1} J_{0},
$$

and we observe the Gauss law of Chern-Simons dynamics is modified by the function $\omega_{1}(|\phi|)$ such that now the conserved charge associated with the $U(1)$ global symmetry is given by

$$
Q=\int d^{2} x e \omega_{1} J^{0}
$$

however as it happens in usual CSH model, the electric charge is nonnull and proportional to the magnetic flux:

$$
Q=\kappa \int d^{2} x B=\kappa \Phi .
$$

Therefore, independently of the functional form of the generalizing functions $\omega_{1}(|\phi|)$ and $\omega(|\phi|)$, the solutions always will be electrically charged.

Likewise, the Ampère law reads

$$
\frac{\kappa}{2} \epsilon^{k \alpha \beta} F_{\alpha \beta}+e \omega J_{k}=0
$$

Along the remaining of the paper, we are interested in time-independent soliton solutions that ensure the finiteness of action (3). These are the stationary points of the energy for which the static

$$
\begin{aligned}
E & =\int d^{2} x\left[-\kappa A_{0} B-e^{2} \omega_{1} A_{0}^{2}|\phi|^{2}+\omega\left|D_{i} \phi\right|^{2}\right. \\
& +V(|\phi|)] .
\end{aligned}
$$

From the statitic Gauss law, we obtain the relation

$$
A_{0}=-\frac{\kappa}{2 e} \frac{B}{\omega_{1}|\phi|^{2}},
$$

which when substituted in (12) leads to the following expression for the energy:

$$
E=\int d^{2} x\left[\frac{\kappa^{2}}{4 e^{2}} \frac{B^{2}}{\omega_{1}|\phi|^{2}}+\omega\left|D_{i} \phi\right|^{2}+V(|\phi|)\right] .
$$

To proceed, we need the fundamental identity

$$
\left|D_{i} \phi\right|^{2}=\left|D_{ \pm} \phi\right|^{2} \pm e B|\phi|^{2} \pm \frac{1}{2} \epsilon_{i k} \partial_{i} J_{k},
$$

where $D_{ \pm} \phi=D_{1} \phi \pm i D_{2} \phi$. Then, by using (15), we may rewrite the energy (14) as

$$
\begin{aligned}
E & =\int d^{2} x\left[\frac{\kappa^{2}}{4 e^{2}} \frac{B^{2}}{\omega_{1}|\phi|^{2}}+V(|\phi|)+\omega\left|D_{ \pm} \phi\right|^{2}\right. \\
& \left. \pm e \omega B|\phi|^{2} \pm \frac{1}{2} \omega \epsilon_{i k} \partial_{i} J_{k}\right] .
\end{aligned}
$$


We observe that the function $\omega(|\phi|)$ in the term $\omega \epsilon_{i k} \partial_{i} J_{k}$ precludes us from implementing the BPS procedure; that is, the integrand must be expressed like a sum of squared terms plus a total derivative plus a term proportional to the magnetic field. Therefore, the key question is about the functional form of $\omega(|\phi|)$ allowing a well defined implementation of the BPS formalism. We start the searching of the function $\omega(|\phi|)$ from the following expression:

$$
\epsilon_{i k} \partial_{i}\left(\omega J_{k}\right)=\omega \epsilon_{i k} \partial_{i} J_{k}+\epsilon_{i k}\left(\partial_{i} \omega\right) J_{k} .
$$

By manipulating the last term $\epsilon_{i k}\left(\partial_{i} \omega\right) J_{k}$ it reads

$$
\epsilon_{i k}\left(\partial_{i} \omega\right) J_{k}=\frac{\partial \omega}{\partial|\phi|^{2}}\left(\partial_{i}|\phi|^{2}\right) \epsilon_{i k} J_{k},
$$

where we have used the fact of $\omega$ being an explicit function of $|\phi|^{2}$. After some algebra the term $\epsilon_{i k}\left(\partial_{i} \omega\right) J_{k}$ becomes

$$
\epsilon_{i k}\left(\partial_{i} \omega\right) J_{k}=|\phi|^{2} \frac{\partial \omega}{\partial|\phi|^{2}} \epsilon_{i k} \partial_{i} J_{k}+2 e B|\phi|^{4} \frac{\partial \omega}{\partial|\phi|^{2}} .
$$

Substituting this equation in (17) we arrive to

$$
\begin{aligned}
\epsilon_{i k} \partial_{i}\left(\omega J_{k}\right)= & \left(\omega+|\phi|^{2} \frac{\partial \omega}{\partial|\phi|^{2}}\right) \epsilon_{i k} \partial_{i} J_{k} \\
& +2 e B|\phi|^{4} \frac{\partial \omega}{\partial|\phi|^{2}} .
\end{aligned}
$$

Here we impose that the function $\omega$ satisfies the following equation:

$$
\omega+|\phi|^{2} \frac{\partial \omega}{\partial|\phi|^{2}}=\beta \omega,
$$

with $\beta$ being a real constant. By solving (21) we obtain the explicit functional form of $\omega(|\phi|)$,

$$
\omega=C|\phi|^{2 \beta-2},
$$

where the constant $C$ adjusts conveniently the mass dimension of $\omega$.

The key condition (21) allows rewriting (20) in a more simplified form

$$
\epsilon_{i k} \partial_{i}\left(\omega J_{k}\right)=\beta \omega \epsilon_{i k} \partial_{i} J_{k}+2 e(\beta-1) \omega B|\phi|^{2},
$$

allowing writing the term $\omega \epsilon_{i k} \partial_{i} J_{k}$ in the following way:

$$
\omega \epsilon_{i k} \partial_{i} J_{k}=\frac{1}{\beta} \epsilon_{i k} \partial_{i}\left(\omega J_{k}\right)-2 e \frac{\beta-1}{\beta} \omega B|\phi|^{2} .
$$

By introducing it in (16), the energy becomes

$$
\begin{aligned}
E & =\int d^{2} x\left[\frac{\kappa^{2}}{4 e^{2}} \frac{B^{2}}{\omega_{1}|\phi|^{2}}+V(|\phi|)+\omega\left|D_{ \pm} \phi\right|^{2}\right. \\
& \left. \pm \frac{1}{\beta} e \omega B|\phi|^{2} \pm \frac{1}{2 \beta} \epsilon_{i k} \partial_{i}\left(\omega J_{k}\right)\right] .
\end{aligned}
$$

We write the two first terms as

$$
\begin{aligned}
\frac{\kappa^{2}}{4 e^{2}} \frac{B^{2}}{|\phi|^{2} \omega_{1}}+V= & \frac{\kappa^{2}}{4 e^{2}} \frac{1}{|\phi|^{2} \omega_{1}}\left(B \mp \frac{2 e}{\kappa}|\phi| \sqrt{\omega_{1} V}\right)^{2} \\
& \pm \frac{\kappa B}{e|\phi|} \sqrt{\frac{V}{\omega_{1}}} .
\end{aligned}
$$

By substituting in (25), we have

$$
\begin{aligned}
E & =\int d^{2} x\left[\frac{\kappa^{2}}{4 e^{2}} \frac{1}{|\phi|^{2} \omega_{1}}\left(B \mp \frac{2 e|\phi|}{\kappa} \sqrt{\omega_{1} V}\right)^{2}\right. \\
& +\omega\left|D_{ \pm} \phi\right|^{2} \pm \frac{1}{2 \beta} \epsilon_{i k} \partial_{i}\left(\omega J_{k}\right) \\
& \left. \pm B\left(\frac{\kappa}{e|\phi|} \sqrt{\frac{V}{\omega_{1}}}+\frac{1}{\beta} e \omega|\phi|^{2}\right)\right] .
\end{aligned}
$$

To finish the BPS procedure, we observe that if in the third row the term multiplying to the magnetic field is equal to $e v^{2}$, it allows defining explicitly the form of the potential $V(|\phi|)$,

$$
V(|\phi|)=\frac{e^{4} v^{4}}{\kappa^{2}} \omega_{1}|\phi|^{2}\left(1-\frac{|\phi|^{2 \beta}}{v^{2 \beta}}\right)^{2},
$$

where we have substituted the explicit form of $\omega(|\phi|)$ given by (22) with $C=\beta v^{2-2 \beta}$ in order for the vacuum expectation value of the Higgs field to be $|\phi|=v$. The function $\omega_{1}(|\phi|)$ still remains arbitrary. Hence, the energy (27) reads

$$
\begin{aligned}
E & =\int d^{2} x\left\{ \pm e v^{2} B \pm \frac{1}{2 \beta} \epsilon_{i k} \partial_{i}\left(\omega J_{k}\right)+\omega\left|D_{ \pm} \phi\right|^{2}\right. \\
& +\frac{\kappa^{2}}{4 e^{2}|\phi|^{2} \omega_{1}}[B \\
& \left.\left.\mp \frac{2 e^{3} v^{2}}{\kappa^{2}} \omega_{1}|\phi|^{2}\left(1-\frac{|\phi|^{2 \beta}}{v^{2 \beta}}\right)\right]^{2}\right\} .
\end{aligned}
$$

We see that under appropriated boundary conditions the total derivative gives null contribution to the energy. Then, the energy is bounded below by a multiple of the magnetic flux magnitude (for positive flux we choose the upper signs, and for negative flux we choose the lower signs):

$$
E \geq \pm e v^{2} \int d^{2} x B=e v^{2}|\Phi| .
$$

This bound is saturated by fields satisfying the Bogomolnyi or self-dual equations [12]

$$
\begin{aligned}
D_{ \pm} \phi & =0, \\
B & = \pm \frac{2 e^{3} v^{2}}{\kappa^{2}} \omega_{1}|\phi|^{2}\left(1-\frac{|\phi|^{2 \beta}}{v^{2 \beta}}\right) .
\end{aligned}
$$


If we require that the magnetic field be nonsingular at origin, the function $\omega_{1}(|\phi|)$ should behave like $|\phi|^{2 \delta}$ with $\delta \geq-1$. On the other hand, positivity and finiteness of the BPS energy density require $\beta \geq 1$.

Below we study interesting models by given a specific form of the functions $\omega$ and $\omega_{1}$.

\section{Some Simple Models}

In the following we analyze some interesting but simple models by setting

$$
\begin{aligned}
\omega(|\phi|) & =(N+1) \frac{|\phi|^{2 N}}{v^{2 N}}, \\
\omega_{1}(|\phi|) & =\frac{|\phi|^{2 M}}{v^{2 M}} .
\end{aligned}
$$

The BPS potential (28) reads

$$
V(|\phi|)=\frac{e^{4} v^{6}}{\kappa^{2}} \frac{|\phi|^{2 M+2}}{v^{2 M+2}}\left(1-\frac{|\phi|^{2 N+2}}{v^{2 N+2}}\right)^{2},
$$

and the BPS equation (32) becomes

$$
B= \pm \frac{2 e^{3} v^{4}}{\kappa^{2}} \frac{|\phi|^{2 M+2}}{v^{2 M+2}}\left(1-\frac{|\phi|^{2 N+2}}{v^{2 N+2}}\right) .
$$

Here, it interesting to note that for $N=0$ and $M=0$ the self-duality equations (31) and (35) become the well known Bogomolnyi equations of the Chern-Simons-Higgs theory [8, 9]:

$$
\begin{aligned}
D_{ \pm} \phi & =0, \\
B & = \pm \frac{2 e^{3}}{\kappa^{2}}|\phi|^{2}\left(v^{2}-|\phi|^{2}\right) .
\end{aligned}
$$

In the case where $N=0$ and $M=-1$, we have

$$
\begin{aligned}
D_{ \pm} \phi & =0, \\
B & = \pm \frac{2 e^{3} v^{2}}{\kappa^{2}}\left(v^{2}-|\phi|^{2}\right) .
\end{aligned}
$$

These equations are essentially the Bogomolnyi equations of the Maxwell-Higgs model, whose solutions are the well known Nielsen-Olesen vortices [53]. The difference lies in the fact that, here, our self-dual solitons carry not only magnetic flux, as in the Higgs model, but also $U(1)$ charge. This is a consequence that in our theory the dynamics of gauge field is dictated by a Chern-Simons term instead of a Maxwell term as in Maxwell-Higgs theory. So, for $N=$ 0 and $M=-1$, we obtain self-dual configurations which are mathematically identical to the Nielsen-Olesen ones but differently our solutions have electric charge.
3.1. Vortex Configurations. Specifically, we look for axially symmetric solutions using the standard static vortex Ansatz

$$
\begin{aligned}
\phi & =v g(r) e^{i n \theta}, \\
A_{\theta} & =-\frac{a(r)-n}{e r} .
\end{aligned}
$$

The Ansatz allows expressing the magnetic field as

$$
B=-\frac{a^{\prime}}{e r}
$$

where ' denotes a derivative in relation to the coordinate $r$. Likewise, the BPS equations (31) and (35) are written as

$$
\begin{aligned}
& g^{\prime}= \pm \frac{a g}{r}, \\
& B=-\frac{a^{\prime}}{e r}= \pm \frac{2 e^{3} v^{4}}{\kappa^{2}} g^{2 M+2}\left(1-g^{2 N+2}\right) .
\end{aligned}
$$

These equations are solved considering the profiles $g$ and $a$ are well behaved functions satisfying the following boundary conditions:

$$
\begin{gathered}
g(0)=0, \\
a(0)=n, \\
g(\infty)=1, \\
a(\infty)=0 .
\end{gathered}
$$

The BPS energy density of the model reading from

$$
E_{\mathrm{BPS}}=2 \pi \int d r r \varepsilon_{\mathrm{BPS}}
$$

is given by

$$
\begin{aligned}
\varepsilon_{\mathrm{BPS}}= & \frac{2 e^{4} v^{6}}{\kappa^{2}} g^{2 M+2}\left(1-g^{2 N+2}\right)^{2} \\
& +2 v^{2}(N+1) g^{2 N}\left(\frac{a g}{r}\right)^{2},
\end{aligned}
$$

and the requirement of finite energy density, for all values of the winding number $n$, imposes $N \geq 0$ and $M \geq-1$.

3.2. Checking the Boundary Conditions. We obtain the behavior of the solutions of (40) and (41) in the neighborhood of $r \rightarrow 0$ using power series method:

$$
\begin{aligned}
& g(r)=G_{n} r^{n}-\frac{e^{4} v^{4}\left(G_{n}\right)^{2 M+3} r^{n(2 M+3)+2}}{2 \kappa^{2}(n M+n+1)^{2}}+\cdots \\
& a(r)=n-\frac{e^{4} v^{4}\left(G_{n}\right)^{2 M+2} r^{n(2 M+2)+2}}{\kappa^{2}(n M+n+1)}+\cdots .
\end{aligned}
$$

It verifies the boundary conditions given in (42). 
For $r \rightarrow+\infty$, the behavior of the soliton solutions becomes similar to the Nielsen-Olesen vortices,

$$
\begin{aligned}
& g(r) \sim 1-G_{\infty} \frac{e^{-m_{s} r}}{\sqrt{r}} \\
& a(r) \sim G_{\infty} m_{s} \sqrt{r} e^{-m_{s} r},
\end{aligned}
$$

where $G_{\infty}$ is a numerical constant determined numerically and $m_{s}$, the self-dual mass of the bosonic fields, is given by

$$
m_{s}=\frac{2 e^{2} v^{2}}{\kappa} \sqrt{N+1} .
$$

It is verified that, for $N=0$, the mass scale is exactly the one of the Chern-Simons-Higgs model:

$$
V(|\phi|)=\frac{e^{4} v^{6}}{\kappa^{2}} \frac{|\phi|^{2 M+2}}{v^{2 M+2}}\left(1-\frac{|\phi|^{2 N+2}}{v^{2 N+2}}\right)^{2}
$$

3.3. Numerical Analysis. Below, without loss of generality we set $e=1, v=1, \kappa=1$.

Before performing the numerical solution of the self-dual equations (40) and (41) we do the following observations in relation to the BPS potential (34). First, it provides a $|\phi|^{4}$ potential for $M=-1$ and $N=0$,

$$
V(|\phi|)=\left(1-|\phi|^{2}\right)^{2}
$$

Second, the BPS potential also provides a family of $|\phi|^{6}$ potentials when the condition $M=-2 N$ is satisfied and $N$ is restricted to the interval $0 \leq N \leq 1 / 2$,

$$
V(|\phi|)=|\phi|^{-4 N+2}\left(1-|\phi|^{2 N+2}\right)^{2} .
$$

Below, our numerical analysis considers only these two potentials to solve the BPS equations (40) and (41). In particular, we solve the Bogomolnyi equations only for winding number $n=1$.

In the figures, the red line represents the case where $M=-1$ and $N=0$ providing the Nielsen-Olesen-like vortices, whereas the blue lines depict the vortex solutions for the values of $M$ and $N$ generating some $|\phi|^{6}$ potentials. In particular, we have plotted three solutions in blue lines:

(i) $M=0$ and $N=0$, which generates the well known Chern-Simons-Higgs vortices.

(ii) $M=-0.5$ and $N=0.25$, associated to the self-dual potential

$$
V(|\phi|)=|\phi|\left(1-|\phi|^{5 / 2}\right)^{2}
$$

(iii) $M=-1$ and $N=0.5$, associated to the self-dual potential

$$
V(|\phi|)=\left(1-|\phi|^{3}\right)^{2}
$$

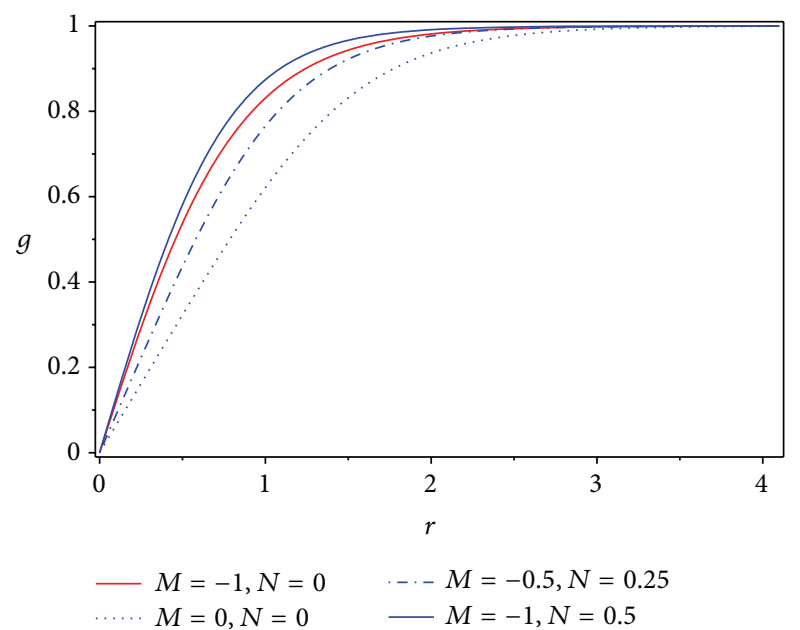

Figure 1: The profiles of the Higgs field $g(r)$ for $n=1$. The red lines represent the solutions for a $|\phi|^{4}$ potential and blue lines for $|\phi|^{6}$ potentials.

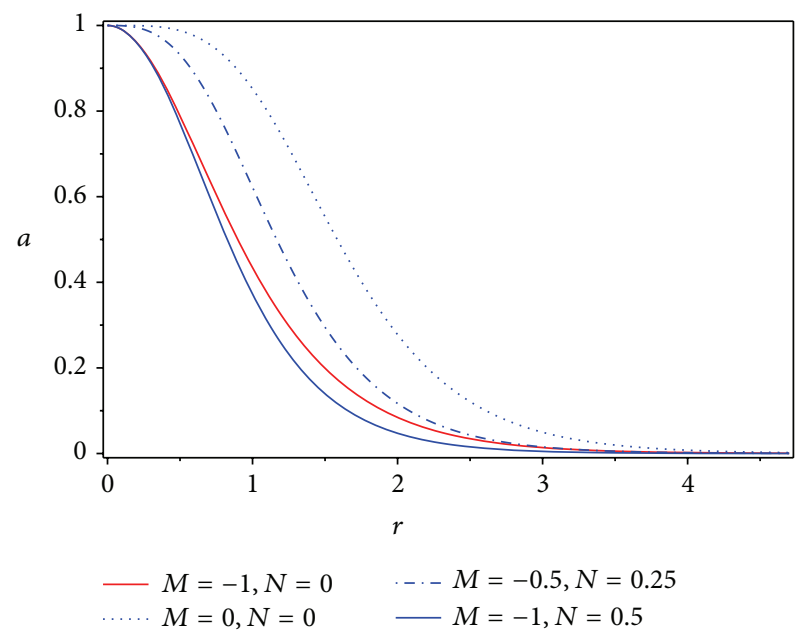

Figure 2: The profiles of the gauge field $a(r)$ for $n=1$. The red lines represent the solutions for a $|\phi|^{4}$ potential and blue lines for $|\phi|^{6}$ potentials.

Note that in the cases $M=-1, N=0$ and $M=-1$, $N=0.5$, that is, the cases associated with the potentials (50) and (53), there is only one degenerate vacuum at $|\phi|=1$. This fact leads us to similar solutions, which can be appreciated in Figures 1, 2, 4, and 3.

For the cases where $M \neq-1$, the $|\phi|^{6}$ potential has two vacua: $|\phi|=0$ and $|\phi|=1$. In these cases, the profiles of the magnetic field are rings whose maximum amplitude, for increasing values of $N$, approaches the origin (see Figure 4). Also the profiles of the BPS energy density have a ring-like format (see Figure 5) and the ring format is explicit for $n>1$.

On the other hand, for electric field, whenever the values of $M$ and $N$ here are considered, the profiles always are rings around the origin (see Figure 3 ). 


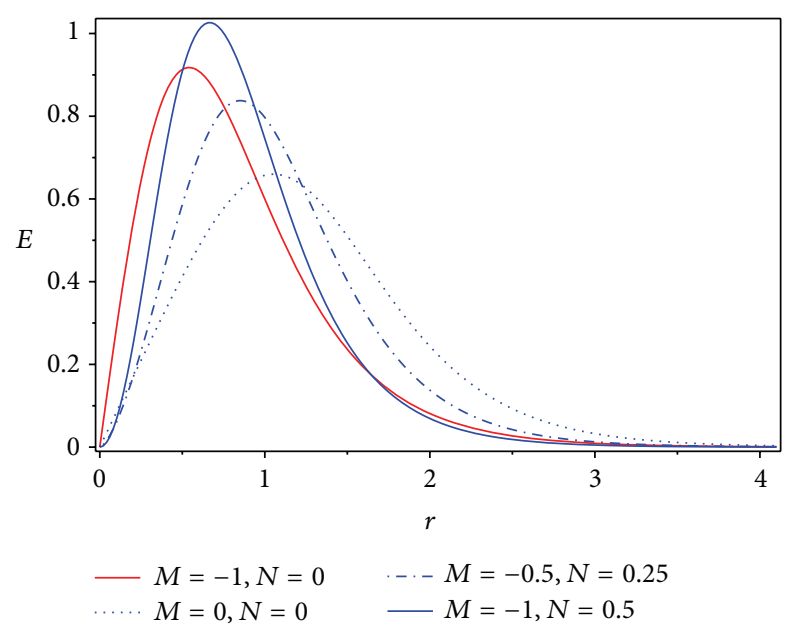

FIgURE 3: The profiles of the electric field $E(r)=A_{0}^{\prime}(r)$ for $n=1$. The red lines represent the solutions for a $|\phi|^{4}$ potential and blue lines for $|\phi|^{6}$ potentials.

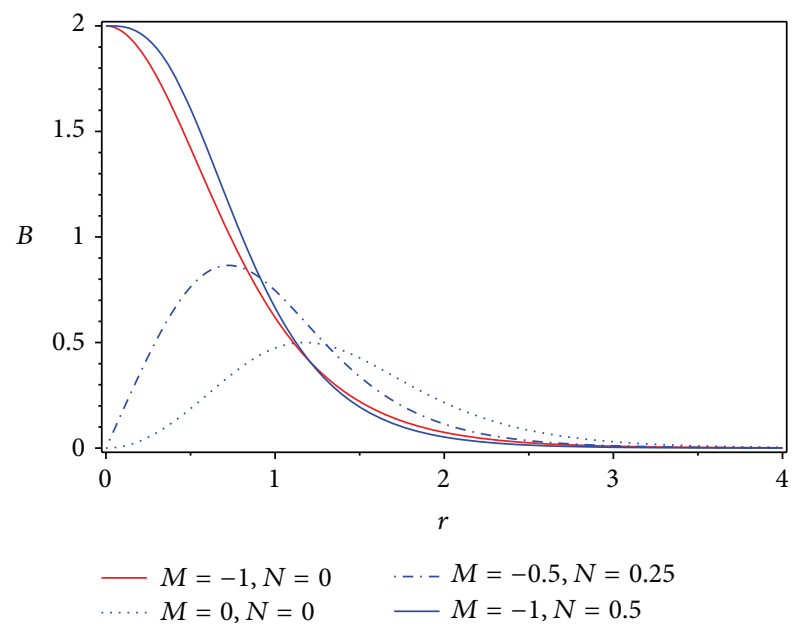

FIgURE 4: The profiles of the magnetic field $B(r)$ for $n=1$. The red lines represent the solutions for a $|\phi|^{4}$ potential and blue lines for $|\phi|^{6}$ potentials.

\section{Remarks and Conclusions}

In summary, we have proposed a generalized Abelian Chern-Simons-Higgs model with explicit breaking of Lorentz covariance and explored the respective Bogomolnyi framework. During the implementation of the BPS trick it is shown that the generalized functions should satisfy some requirements: The function $\omega(|\phi|)$ must be a monomial, that is, $\omega=C|\phi|^{2 \beta-2}$ for all $\beta \geq 1$, and the function $\omega_{1}(|\phi|)$ must be regular at the origin $\left(\omega_{1} \propto|\phi|^{2 \delta}\right.$ with $\left.\delta \geq-1\right)$. Under such conditions imposed on the generalized functions, the existence of self-dual solitonic configurations satisfying Bogomolnyi equations whose magnetic field and BPS energy density are well behaved is guaranteed. As we expected, the infinity family of self-dual configurations have finite energy which is proportional to the magnitude of the magnetic flux. In particular, we have studied the self-dual vortices provided

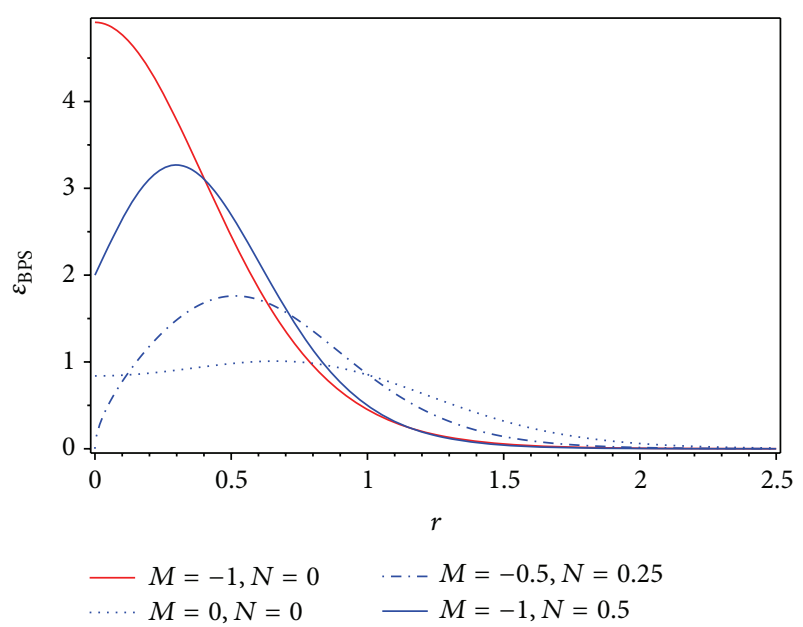

FIGURE 5: The profiles of the BPS energy density $\varepsilon_{\text {BPS }}(r)$ for $n=1$. The red lines represent the solutions for a $|\phi|^{4}$ potential and blue lines for $|\phi|^{6}$ potentials.

by the choice of $\omega_{1}(|\phi|)=(N+1)|\phi|^{2 N}$ and $\omega_{1}(|\phi|)=|\phi|^{2 M}$. It was shown the vortex solutions of the Maxwell-Higgs model and the Chern-Simons-Higgs model can be also obtained. Besides that, we have constructed two new solitonic solutions which correspond to Chern-Simons theory coupled to two types of $|\phi|^{6}$ potentials given by (52) and (53), respectively.

Finally, it is worthwhile to point out that existence of BPS states is linked to the existence of an $\mathcal{N}=2$ extended supersymmetric model [54]. We are studying such a possibility despite the fact that in this model the Lorentz symmetry is explicitly broken. Advances in this direction will be reported elsewhere.

\section{Competing Interests}

The authors declare that there is no conflict of interests regarding the publication of this paper.

\section{Acknowledgments}

Rodolfo Casana acknowledge CNPq, CAPES, and FAPEMA (Brazilian agencies) for financial support. Lucas Sourrouille is supported by CONICET.

\section{References}

[1] S. K. Paul and A. Khare, "Charged vortices in an abelian Higgs model with Chern-Simons term," Physics Letters B, vol. 174, no. 4, pp. 420-422, 1986, Erratum in: Physics Letters B, vol. 177, p. $453,1986$.

[2] H. J. de Vega and F. A. Schaposnik, "Vortices and electrically charged vortices in non-Abelian gauge theories," Physical Review D, vol. 34, no. 10, pp. 3206-3213, 1986.

[3] R. Jackiw and S. Y. Pi, "Self-dual chern-simons solitons," Progress of Theoretical Physics Supplement, no. 107, pp. 1-40, 1992.

[4] G. Dunne, Self-Dual Chern-Simons Theories, vol. 36 of Lecture Notes in Physics Monographs, 1995. 
[5] G. V. Dunne, "Aspects of Chern-Simons theory," https://arxiv .org/abs/hep-th/9902115.

[6] F. A. Schaposnik, "Vortices," https://arxiv.org/abs/hep-th/ 0611028.

[7] P. A. Horvathy and P. Zhang, "Vortices in (abelian) ChernSimons gauge theory," Physics Reports, vol. 481, no. 5-6, pp. 83$142,2009$.

[8] R. Jackiw and E. J. Weinberg, "Self-dual Chern-Simons vortices," Physical Review Letters, vol. 64, no. 19, pp. 2234-2237, 1990.

[9] J. Hong, Y. Kim, and P. Y. Pac, "Multivortex solutions of the abelian Chern-Simons-Higgs theory," Physical Review Letters, vol. 64 , no. 19, pp. 2230-2233, 1990.

[10] R. Jackiw and S.-Y. Pi, "Soliton solutions to the gauged nonlinear Schrödinger equation on the plane," Physical Review Letters, vol. 64, no. 25, pp. 2969-2972, 1990.

[11] R. Jackiw and S. Y. Pi, "Classical and quantal nonrelativistic Chern-Simons theory," Physical Review D, vol. 42, no. 10, pp. 3500-3513, 1990, Erratum in: Physical Review D, vol. 48, p. 3929, 1993.

[12] E. Bogomol'nyi, "The stability of classical solutions," Soviet Journal of Nuclear Physics, vol. 24, no. 4, pp. 449-454, 1976.

[13] H. J. de Vega and F. A. Schaposnik, "Classical vortex solution of the Abelian Higgs model," Physical Review D, vol. 14, no. 4, pp. 1100-1106, 1976.

[14] C. Lee, K. Lee, and E. J. Weinberg, "Supersymmetry and selfdual Chern-Simons systems," Physics Letters B, vol. 243, no. 1-2, pp. 105-108, 1990.

[15] R. Jackiw, K.-M. Lee, and E. J. Weinberg, "Self-dual ChernSimons solitons," Physical Review D, vol. 42, no. 10, pp. 34883499, 1990.

[16] T. H. R. Skyrme, "Particle states of a quantized meson field," Proceedings of the Royal Society of London A, vol. 262, no. 1309, pp. 237-245, 1961.

[17] C. Armendáriz-Picón, T. Damour, and V. Mukhanov, " $k$ inflation," Physics Letters B, vol. 458, no. 2-3, pp. 209-218, 1999.

[18] C. Armendariz-Picon, V. Mukhanov, and P. J. Steinhardt, "Dynamical solution to the problem of a small cosmological constant and late-time cosmic acceleration," Physical Review Letters, vol. 85, no. 21, pp. 4438-4441, 2000.

[19] C. Armendariz-Picon, V. Mukhanov, and P. J. Steinhardt, "Essentials of k-essence," Physical Review D, vol. 63, Article ID 103510, 2001.

[20] T. Chiba, T. Okabe, and M. Yamaguchi, "Kinetically driven quintessence," Physical Review D, vol. 62, Article ID 023511, 2000.

[21] M. Malquarti, E. J. Copeland, and A. R. Liddle, " $k$-essence and the coincidence problem," Physical Review D, vol. 68, no. 2, Article ID 023512, 2003.

[22] J. U. Kang, V. Vanchurin, and S. Winitzki, "Attractor scenarios and superluminal signals in $k$-essence cosmology," Physical Review D, vol. 76, Article ID 083511, 2007.

[23] E. Babichev, V. Mukhanov, and A. Vikman, " $k$-essence, superluminal propagation, causality and emergent geometry," Journal of High Energy Physics, vol. 2008, no. 2, article 101, 45 pages, 2008.

[24] A. Sen, “Tachyon matter," Journal of High Energy Physics, no. 7, article 065, 2002.

[25] N. Arkani-Hamed, H.-C. Cheng, M. A. Luty, and S. Mukohyama, "Ghost condensation and a consistent infrared modification of gravity," Journal of High Energy Physics, vol. 2004, no. 5, article 074, 2004.
[26] N. Arkani-Hamed, P. Creminelli, S. Mukohyama, and M. Zaldarriaga, "Ghost inflation," Journal of Cosmology and Astroparticle Physics, vol. 2004, no. 4, p. 1, 2004.

[27] S. L. Dubovsky, "Star tracks in the ghost condensate," Journal of Cosmology and Astroparticle Physics, vol. 2004, no. 7, p. 9, 2004.

[28] D. Krotov, C. Rebbi, V. Rubakov, and V. Zakharov, "Holes in the ghost condensate," Physical Review D, vol. 71, no. 4, Article ID 045014, 2005.

[29] A. Anisimov and A. Vikman, "The classical stability of the ghost condensate," Journal of Cosmology and Astroparticle Physics, vol. 2005, Article ID 0504, 2005.

[30] V. Mukhanov and A. Vikman, "Enhancing the tensor-to-scalar ratio in simple inflation," Journal of Cosmology and Astroparticle Physics, vol. 2006, no. 2, p. 4, 2006.

[31] C. Armendariz-Picon and E. A. Lim, "Haloes of k-essence," Journal of Cosmology and Astroparticle Physics, vol. 2005, no. 8, article 007, 2005.

[32] D. Bazeia, E. da Hora, C. dos Santos, and R. Menezes, "Generalized self-dual Chern-Simons vortices," Physical Review D, vol. 81, no. 12, Article ID 125014, 2010.

[33] D. Bazeia, E. da Hora, R. Menezes, H. P. de Oliveira, and C. dos Santos, "Compactlike kinks and vortices in generalized models," Physical Review D, vol. 81, no. 12, Article ID 125016, 2010.

[34] C. dos Santos and E. da Hora, "Domain walls in a generalized Chern-Simons model," The European Physical Journal C, vol. 70, no. 4, pp. 1145-1151, 2010.

[35] C. dos Santos and E. da Hora, "Lump-like solitons in a generalized Abelian-Higgs Chern-Simons model," The European Physical Journal C, vol. 71, article 1519, 2011.

[36] C. dos Santos, "Compact solitons in an Abelian-Higgs ChernSimons model," Physical Review D, vol. 82, Article ID 125009, 2010.

[37] D. Bazeia, E. da Hora, C. dos Santos, and R. Menezes, "BPS solutions to a generalized Maxwell-Higgs model," The European Physical Journal C, vol. 71, article 1833, 2011.

[38] D. Bazeia, R. Casana, E. da Hora, and R. Menezes, "Generalized self-dual Maxwell-Chern-Simons-Higgs model," Physical Review D, vol. 85, no. 12, Article ID 125028, 2012.

[39] R. Casana, M. M. Ferreira Jr., and E. da Hora, "Generalized BPS magnetic monopoles," Physical Review D, vol. 86, no. 8, Article ID 085034, 2012.

[40] E. Babichev, “Global topological k-defects," Physical Review. D. Third Series, vol. 74, no. 8, Article ID 085004, 2006.

[41] E. Babichev, “Gauge k-vortices," Physical Review D, vol. 77, Article ID 065021, 2008.

[42] C. Adam, J. Sanchez-Guillen, and A. Wereszczynski, " $k$-defects as compactons," Journal of Physics A, vol. 40, no. 45, pp. 1362513643, 2007, Erratum-ibid. vol. 42, Article ID 089801, 2009.

[43] C. Adam, N. Grandi, J. Sanchez-Guillen, and A. Wereszczynski, " $K$ fields, compactons and thick branes," Journal of Physics A, vol. 41, no. 21, Article ID 212004, 2008, Erratum in: Journal of Physics A, vol. 42, Article ID 159801, 2009.

[44] C. Adam, N. Grandi, P. Klimas, J. Sánchez-Guillén, and A. Wereszczyński, "Compact self-gravitating solutions of quartic $(K)$ fields in brane cosmology," Journal of Physics A: Mathematical and Theoretical, vol. 41, no. 37, Article ID 375401, 2008.

[45] C. Adam, P. Klimas, J. Sánchez-Guillén, and A. Wereszczyński, "Compact gauge K vortices," Journal of Physics A: Mathematical and Theoretical, vol. 42, no. 13, Article ID 135401, 2009. 
[46] L. Sourrouille, "Galilean symmetry in generalized Abelian Schrödinger-Higgs models with and without gauge field interaction," Modern Physics Letters A, vol. 30, no. 39, Article ID 1501211, 13 pages, 2015.

[47] R. Casana and L. Sourrouille, "Self-dual soliton solutions in a Chern-Simons-CP(1) model with a nonstandard kinetic term," Modern Physics Letters A, vol. 29, no. 23, Article ID 1450124, 2014.

[48] L. Sourrouille, "Charged nielsen-olesen vortices from a generalized abelian chern-simons-higgs theory," Physical Review D, vol. 87, no. 6, Article ID 067701, 2013.

[49] L. Sourrouille, "Self-dual soliton solution in a generalized Jackiw-Pi model," Physical Review D, vol. 86, Article ID 085014, 2012.

[50] E. Babichev, "Global topological $k$-defects," Physical Review D, vol. 74, no. 8, Article ID 085004, 7 pages, 2006.

[51] D. Bazeia, L. Losano, R. Menezes, and J. C. R. E. Oliveira, "Generalized global defect solutions," European Physical Journal C, vol. 51, no. 4, pp. 953-962, 2007.

[52] X.-H. Jin, X.-Z. Li, and D.-J. Liu, "A gravitating global kmonopole," Classical and Quantum Gravity, vol. 24, no. 11, pp. 2773-2780, 2007.

[53] H. B. Nielsen and P. Olesen, "Vortex-line models for dual strings," Nuclear Physics B, vol. 61, pp. 45-61, 1973.

[54] E. Witten and D. Olive, "Supersymmetry algebras that include topological charges," Physics Letters B, vol. 78, no. 1, pp. 97-101, 1978. 

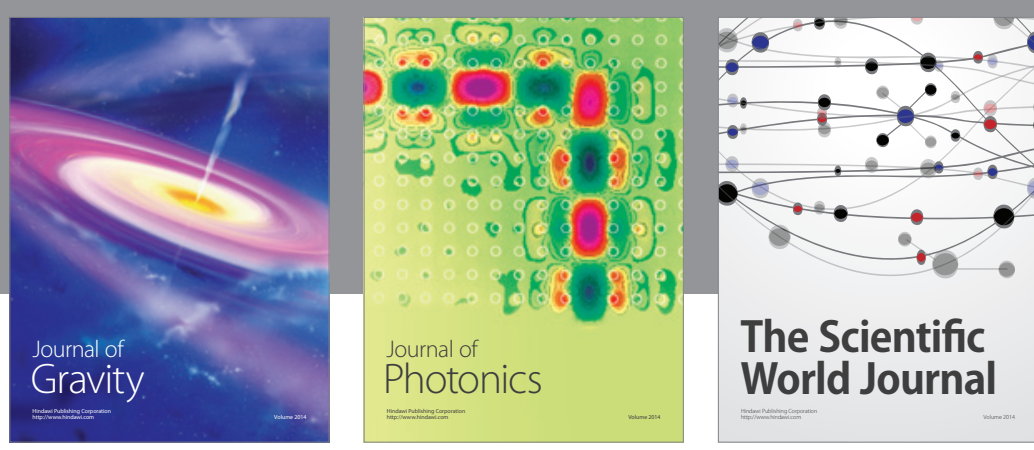

The Scientific World Journal
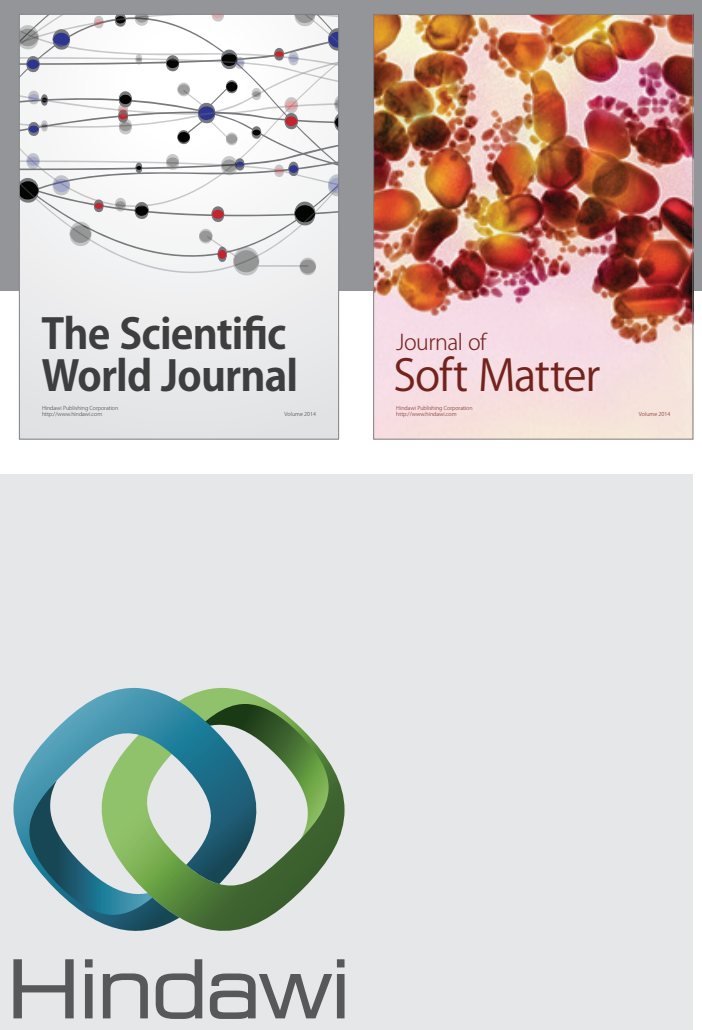

Submit your manuscripts at

http://www.hindawi.com

nternational Journal of

Statistical Mechanics
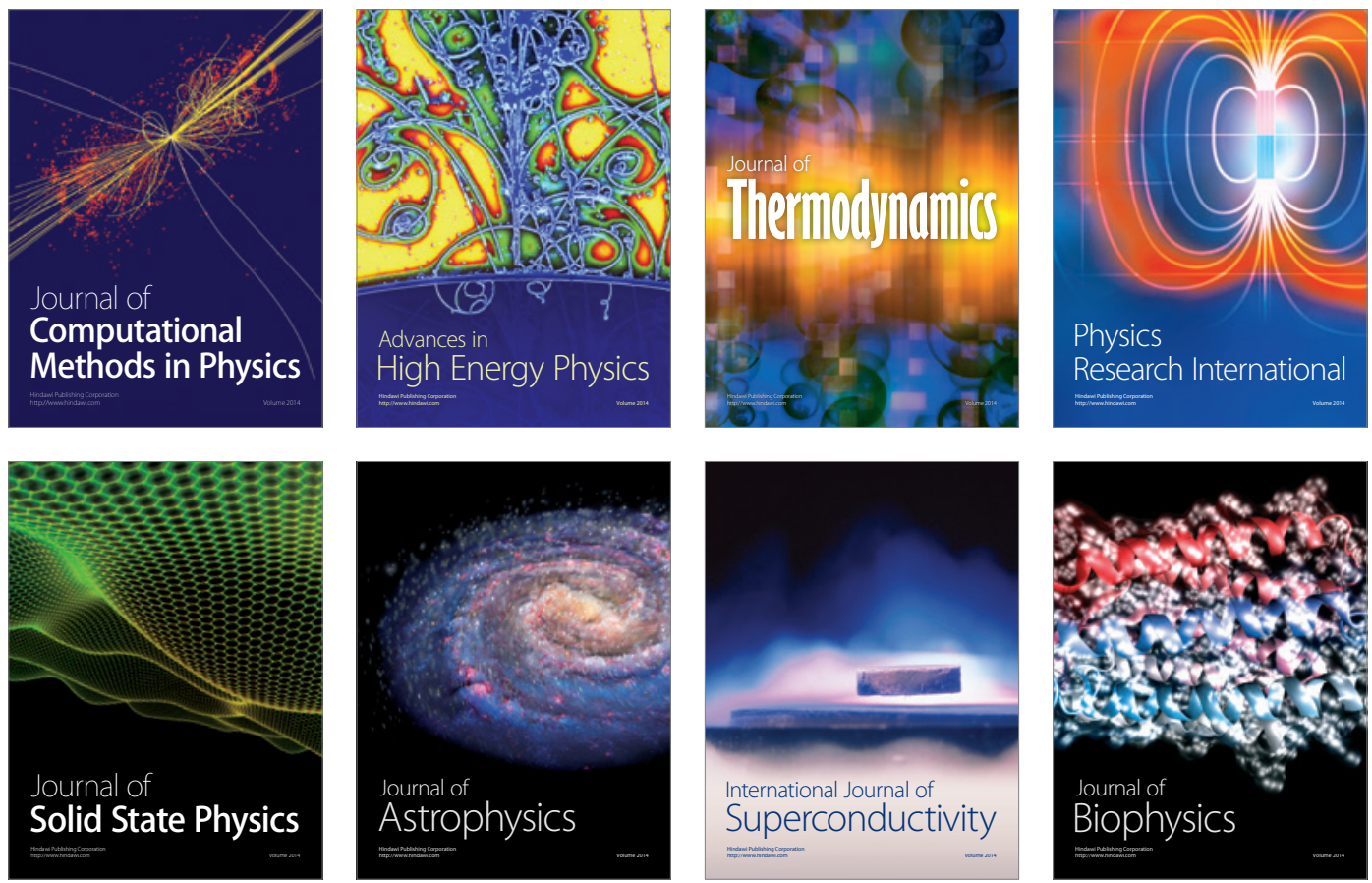
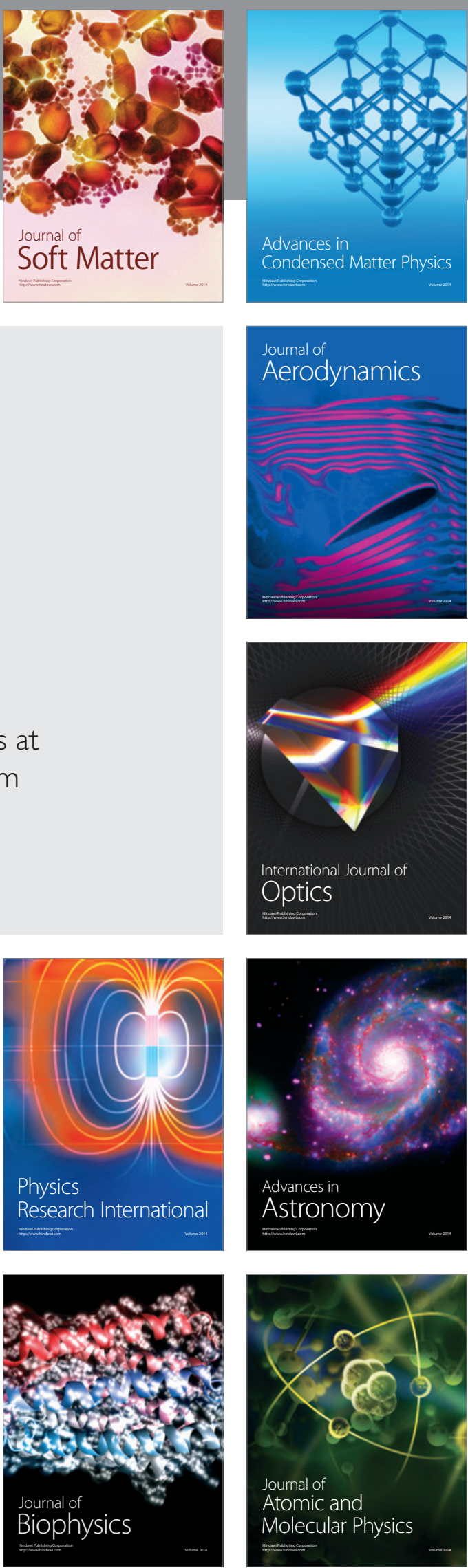